\title{
Improvement in Response Speed for Bistable Light Switching of (Liquid Crystalline Polymer/Low Molecular Weight Liquid Crystals) Ternary Composite System in Induced Smectic State
}

\author{
Jenn Chiu Hwang, ${ }^{\dagger}$ Hirotsugu KiKuchI, and Tisato KaJIYAma ${ }^{\dagger \dagger}$ \\ Department of Chemical Science and Technology, Faculty of Engineering, \\ Kyushu University, 6-10-1 Hakozaki, Higashi-ku, Fukuoka 812, Japan
}

(Received September 30, 1994)

\begin{abstract}
Bistable light switching characteristics of the composite system composed of side chain type liquid crystalline polymer (LCP) and two kinds of low molecular weight liquid crystals (LCs) have been investigated. Each component was in a nematic state but the ternary composite showed an induced smectic phase over the wide ranges of both mixing concentration and temperature. The reversible turbid (light scattering)-transparent switching was observed upon the application of electric fields with low and high frequencies, respectively. Both transparent and turbid states of the ternary composite could be maintained stably, even though an electric field was turned off. The ternary composite system exhibited novel characteristics on bistable and reversible light switching. The response speed for bistable light switching could be remarkably improved by reducing the LCP fraction, maintaining an induced smectic state. Then, a novel type of the (LCP/LCs) composite system with a short response time at room temperature was realized for that with bistable and reversible light switching.

KEY WORDS Liquid Crystalline Polymer / Induced Smectic Phase / Light

Scattering / Bistable and Reversible Light Switching / Memory Effect /
\end{abstract}

The preparation methods and the novel permselective characteristics of (polymer/LC) composite films have been extensively reported. ${ }^{1-3}$ Also, various types of (polymer/LC) composite systems have been reported as large area and flexible light-intensity controllable films. ${ }^{4-11}$ Since thermotropic liquid crystalline polymers (LCPs) with mesogenic side chain groups exhibit both inherent mesomorphic properties of LC and excellent mechanical characteristics of polymeric materials, LCPs have attracted a major attention due to their promising applications as electro-optical devices. ${ }^{12}$ However, since LCPs in a mesophase state are more viscous than LCs, the response time of LCPs to an external stimulation such as an electric or a magnetic field is fairly longer than that of LCs. A (LCP/LC) mixture in which LC takes a role of solvent or diluent to LCP has been studied in order to reduce the magnitude of viscosity of LCP, in other words, to reduce the magnitude of response time for the LCP. ${ }^{13-16}$

It was reported that the binary mixtures of LCs with a strong polar cyano or nitro terminal group and a weak polar one induced a smectic phase (ISP). ${ }^{17-20}$ This concept was applied to the binary mixture of nematic LCP with a weak polar end group in the side chain and nematic LC with a strong polar end group, and the binary mixture also exhibited an induced smectic phase. ${ }^{21-23}$ Then, the (LCP/LC)

† Present address: Department of Chemical Engineering, Yuan-Tze Institute of Technology, 135 Yuan-Tung Road, Nei-Li, Taoyuan Shian 32026, Taiwan, R. O. C.

${ }^{\dagger \dagger}$ To whom correspondence should be addressed. 
mixture in an induced smectic state is expected as a novel type of "light valve" exhibiting bistable and reversible light switching characteristics, that is, a memory effect.

In this paper, the phase transition behaviors, aggregation states, and also, a bistable and reversible light switching based on light scattering have been investigated for the ternary composite system in an induced smectic state, being composed of the side chain type LCP and two kinds of LCs.

\section{EXPERIMENTAL}

\section{Materials}

The chemical structures of LCP and LCs are shown in Figure 1. The side chain type LCP (PS3EM) is polymethylsiloxane with a weak polar terminal group in a mesogenic side group. The molecular weight of PS3EM is $1.1 \times 10^{4}$. 5OCB and HPPB were used as LCs. $5 \mathrm{OCB}$ has a strong polar cyano terminal group and HPPB has no such a group. HPPB has the same rigid ester group as the mesogenic side chain group of PS3EM. The mesomorphic phase of LCP and LCs are nematic states. The mixture of PS3EM/HPPB/5OCB was dissolved in an acetone and the film of the ternary composite was prepared by a solvent-casting method.

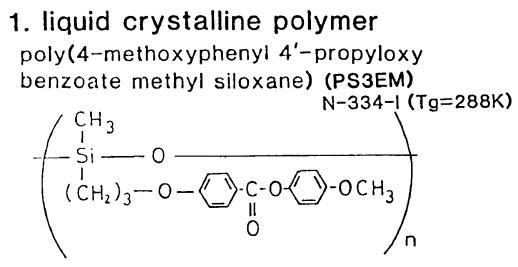

2. liquid crystal

(a) 4-hexyloxyphenyl 4'-pentyl benzoate $\mathrm{C}_{5} \mathrm{H}_{11}-\mathrm{C}_{0}-\mathrm{O}-\mathrm{C}-\mathrm{C}_{6} \mathrm{H}_{13} \quad \begin{aligned} & \text { (HPPB) } \\ & \mathrm{K}-311-\mathrm{N}-334-1\end{aligned}$

(b) 4-cyano 4'-pentyloxy biphenyl (5OCB) $\mathrm{C}_{5} \mathrm{H}_{11}-0-\langle 1-\mathrm{CN} \quad \mathrm{K}-321-\mathrm{N}-341-1$

Figure 1. The chemical structures of liquid crystalline polymer and low molecular weight liquid crystals.

\section{Characterization of the Composite System}

The phase transition behaviors and the aggregation states of the (PS3EM/HPPB/ 5OCB) composite were investigated on the basis of differential scanning calorimetry (DSC), polarizing optical microscopic (POM) observation, and $\mathrm{X}$-ray diffraction studies. The heating and cooling rates for DSC study and POM observation were 5 and $1 \mathrm{~K} \mathrm{~min}^{-1}$, respectively. X-Ray diffraction patterns were taken with a flat plate camera collimated with toroid mirror optics, using Ni-filtered $\mathrm{Cu}-K_{\alpha}$ $(\lambda=0.1542 \mathrm{~nm})$.

\section{Measurement of Electro-Optical Effect}

In order to measure the electro-optical properties, the (PS3EM/HPPB/5OCB) composite was sandwiched between two transparent ITO-glass electrodes, which were separated by the PET spacer of $10 \mu \mathrm{m}$ thick. The surface alignment treatment on the ITO-glass surface was not carried out, so that molecular orientation in the composite was random. The electro-optical effect of the (PS3EM/HPPB/5OCB) composite was studied under the application of a.c. electric field. Transmission intensity of $\mathrm{He}-\mathrm{Ne}$ laser light through the cell was detected with a photodiode and was recorded with a digital storage oscilloscope. The distance between the cell and the photodiode was $305 \mathrm{~mm}$. The measuring temperature for electro-optical switching was controlled with accuracy of $\pm 0.1 \mathrm{~K}$.

\section{RESULTS AND DISCUSSION}

\section{How to Enhance the Response Speed}

In the case that a sufficiently large electric filed, $E$ above the threshold one, $E_{\mathrm{c}}$ is imposed to the (LCP/LC) composite system in a mesophase, the rise time $\tau_{R}$ is written by the following equation, because the composite system is homogeneous (one phase blend). ${ }^{23}$

$$
\tau_{\mathrm{R}}=\eta / \Delta \varepsilon\left(E^{2}-E_{\mathrm{c}}{ }^{2}\right) \approx \eta / \Delta \varepsilon E^{2}
$$

where $\eta$ and $\Delta \varepsilon$ are the viscosity and the 
dielectric anisotropy, respectively. Since the rise time $\tau_{\mathbf{R}}$ is proportional to the magnitude of $\eta$ and the reciprocal of $\Delta \varepsilon$ in the case of the same magnitude of an imposed electric filed $E$, a decrease of viscosity in the (LCP/LC) composite system is apparently effective to increase the rise response speeds for a bistable light switching. Since LCPs in a mesophase state is more viscous than LCs, the magnitude of $\eta$, in general, increases with an increase of the LCP fraction in the (LCP/LC) composite system. Therefore, this indicates that the magnitude of $\eta$ in a mesophase of (LCP/LC) composite system must be reduced in order to realize the faster response speed for a bistable and reversible light switching. As reported for the case of the (smectic LCP/nematic LC) composite in a smectic state, ${ }^{15,16}$ the smectic composite system exhibited a bistable and reversible light switching characteristics upon the application of a.c. electric fields of low and high frequencies. However, the response time was fairly longer at room temperature. Then, the (nematic LCP/nematic LC) composite system in an induced smectic phase was proposed to reduce the response time to several hundreds milliseconds for a bistable and reversible light switching at room temperature. $^{21-23}$

Figure 2 shows the 5OCB molar fraction dependence of the rise response time for the PS3EM/5OCB composite system at a measuring temperature below the nematic-isotropic phase transition temperature, $T_{\mathrm{NI}}$ by $5 \mathrm{~K}$ $\left(T-T_{\mathrm{NI}}=-5 \mathrm{~K}\right)$ as a function of the magnitudes of an applied electric field. When the molar ratio of PS3EM/5OCB was varied from $70 / 30$ to $30 / 70$ in which range the composite exhibited an induced smectic phase, the magnitude of $\tau_{\mathbf{R}}$ decreased a few decades. Since $\triangle \varepsilon^{\prime}$ S of PS3EM and 5OCB are about 1 and 12 , respectively, a change in $\Delta \varepsilon$ of $\mathrm{PS} 3 \mathrm{EM} / 5 \mathrm{OCB}$ when the molar ratio was varied from $70 / 30$ to $30 / 70$ should be at least single decade. Then, such a great change in $\tau_{\mathbf{R}}$ as shown in Figure 2 suggests that the magnitude of $\eta$ is primarily

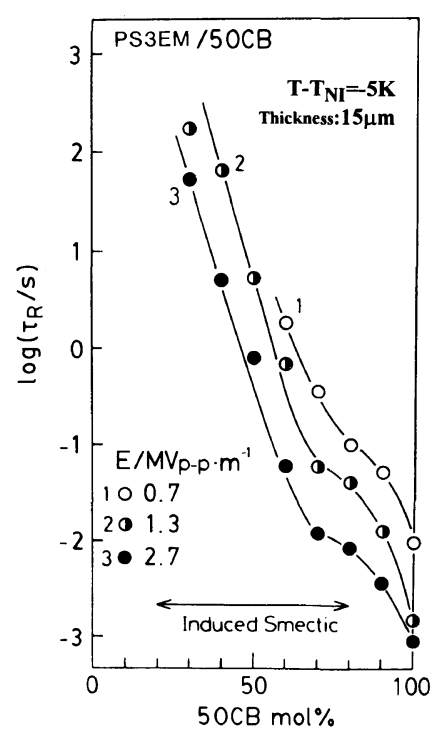

Figure 2. 5OCB molar fraction dependence of rise response time, $\tau_{\mathrm{R}}$ for the (PS3EM/5OCB) composite at $T-T_{\mathrm{NI}}=-5 \mathrm{~K}$ as a function of the magnitude of an applied electric field, $E$.

important to decrease $\tau_{\mathbf{R}}$.

In the case of the (PS3EM/5OCB: $50 / 50$ mol\%) composite system in an induced smectic phase, the composite exhibited very stable memory effect at $T_{\mathrm{NI}}-5 \mathrm{~K}\left(T-T_{\mathrm{NI}}=-5 \mathrm{~K}\right)$ with the magnitude of $\tau_{\mathbf{R}}$ of several hundred milliseconds - a few seconds, depending on the magnitude of an applied electric field as shown in Figure 2. In order to realize the fairly faster response time for the (LCP/LC) composite system, it is apparent that a decrease in the LCP fraction in the composite system is fairly effective, since $\eta$ of LCP is generally higher than that of LC. However, if the ratio of PS3EM/5OCB deviates much from 50/50 in the induced smectic phase, the stability of memory effect is lost. So long as in the binary composite system, it is difficult to realize simultaneously a fast response and a stable memory. Then, the low molecular weight liquid crystal, HPPB was added as the third component to the (PS3EM/ 5OCB) composite system, in order to reduce the molar fraction of LCP, without a change in smectic aggregation state as stable as 
possible. Since HPPB has the corresponding chemical structure to that of the side chain mesogenic group of PS3EM, the (PS3EM/ HPPB) mixture with a weak polar end in the mesogenic side group may become the counter part to 5OCB with a strong polar end to form an induced smectic phase with the fairly low $\eta$. That is, the addition of HPPB can make the molar fraction of PS3EM reduced and also, the magnitude of $\eta$ in the ternary composite system decreased, maintaining similar induced smectic characteristics, such as a memory stability to the (PS3EM/5OCB) composite system.

\section{Phase Transition Behaviors and Aggregation States of the Ternary PS3EM/HPPB/5OCB Composite}

As mentioned above, the (PS3EM/5OCB : $50 / 50 \mathrm{~mol} \%$ ) composite exhibited an excellent bistable and reversible light switching, though the magnitude of $\tau_{R}$ was large at room temperature. Therefore, the molar percentage of the PS3EM/HPPB mixture and 5OCB was maintained to be $50 / 50$. This means that the ratio of $\mathrm{PS} 3 \mathrm{EM} / \mathrm{HPPB}$ is $50-X / X$, when the molar percentage of HPPB is $X(0 \leq X \leq 50)$.

Figure 3 shows the DSC curves of the ternary composite systems. In order to obtain the reproducible DSC data, the 2 nd heating curves were used. The glass transition temperature, $T_{\mathrm{g}}$ of the ternary composite system was observed at around $233-238 \mathrm{~K}$. The $T_{\mathrm{g}}$ of the ternary composite decreased slightly with an addition of HPPB. Also, three endothermic peaks were observed for the crystalline-smectic $(270-278 \mathrm{~K})$, the smectic-nematic (341$344 \mathrm{~K})$, and the nematic-isotropic (346-349 K) phase transitions. Each assignment was discussed in the previous report. ${ }^{16,23}$ The sharpness of each endothermic peak increased gradually with increasing the molar percentage of HPPB from $10 \%$ to $40 \%$. This may be due to a more homogeneous thermal transition order with a decrease in the LCP fraction. Since the endothermic peaks attributed to the phase

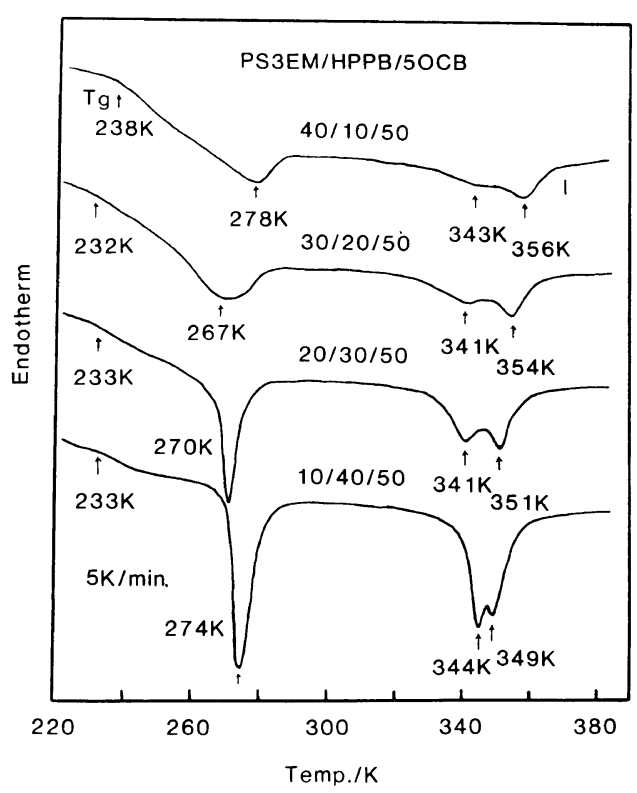

Figure 3. DSC curves of the (PS3EM/HPPB/5OCB : $50-X / X / 50 \mathrm{~mol} \%)$ ternary systems.

transitions of HPPB (K-311-N-334-I) were not observed over a wide fraction range of HPPB, it seems reasonable to conclude that the ternary composite forms a homogeneously mixed mesomorphic phase. This indicates that the ternary composite can be used as a miscible blend sample with a homogeneous mesomorphic phase over a whole concentration range $(0-50 \mathrm{~mol} \%)$ of HPPB.

Figure 4 shows the phase diagram of the (PS3EM/HPPB/5OCB : $50-X / X / 50 \mathrm{~mol} \%$ ) composite, which was obtained on the basis of DSC, POM, and X-ray studies. As mentioned above, the mol\% of (PS3EM + HPPB)/5OCB was kept to be 50/50. The fan-shape texture which is characteristic of a smectic phase was observed under POM for the ternary composite system. A smectic phase was induced over a wide range of both mixing concentration and temperature as shown in Figure 4. Even in the extreme case of the (PS3EM/HPPB/5OCB) composites of $(50 / 0 / 50)$ and $(0 / 50 / 50)$, an induced smectic phase was observed. Then, this indicates that the mol\% of LCP can be reduced 


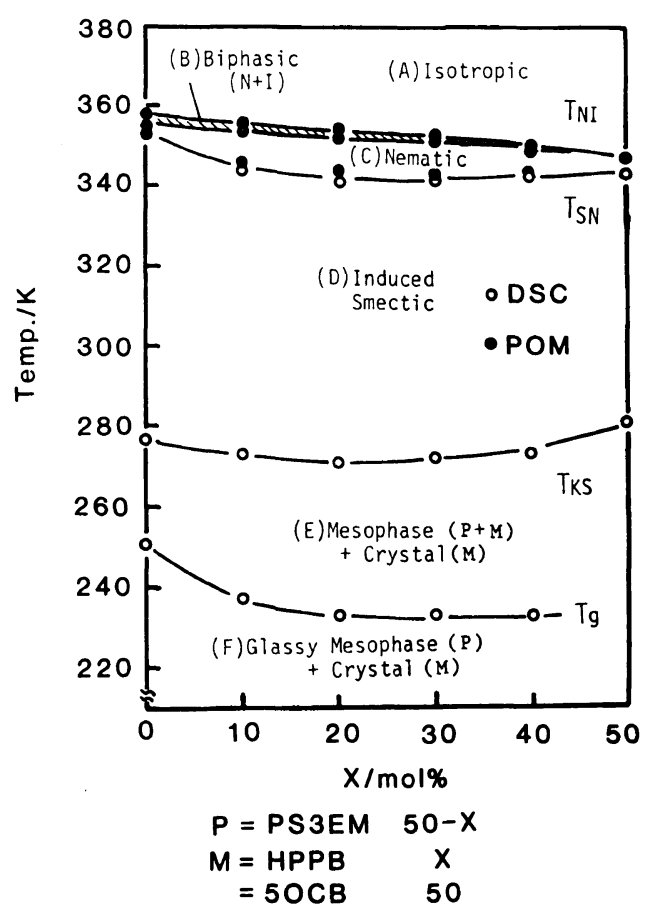

Figure 4. Phase diagram of the (PS3EM/HPPB/5OCB : $50-X / X / 50 \mathrm{~mol} \%)$ ternary composite system.

by mixing of HPPB in the (PS3EM/HPPB/ 5OCB) ternary composite system, maintaining the similar characteristics to an induced smectic phase in the (PS3EM/5OCB:50/50) binary composite. The narrow biphasic nematicisotropic region of about $3 \mathrm{~K}$ wide, in which the nematic and isotropic phase coexisted, was recognized by POM observation.

In order to clarify the formation of an induced smectic phase by mixing the three components, PS3EM, HPPB, and 5OCB of which each mesophase is nematic, $\mathrm{X}$-ray diffraction studies were carried out in a temperature range of an induced smectic phase as shown in Figure 5. Figure 5 shows the X-ray diffraction pattern of the (PS3EM/HPPB/ 5OCB : $30 / 20 / 50 \mathrm{~mol} \%$ ) ternary composite. Sharp Debye rings in a low Bragg angle region which are not observed in the nematic phase clearly indicates the existence of layer structure corresponding to an induced smectic phase, since a reciprocal of these spacings is roughly
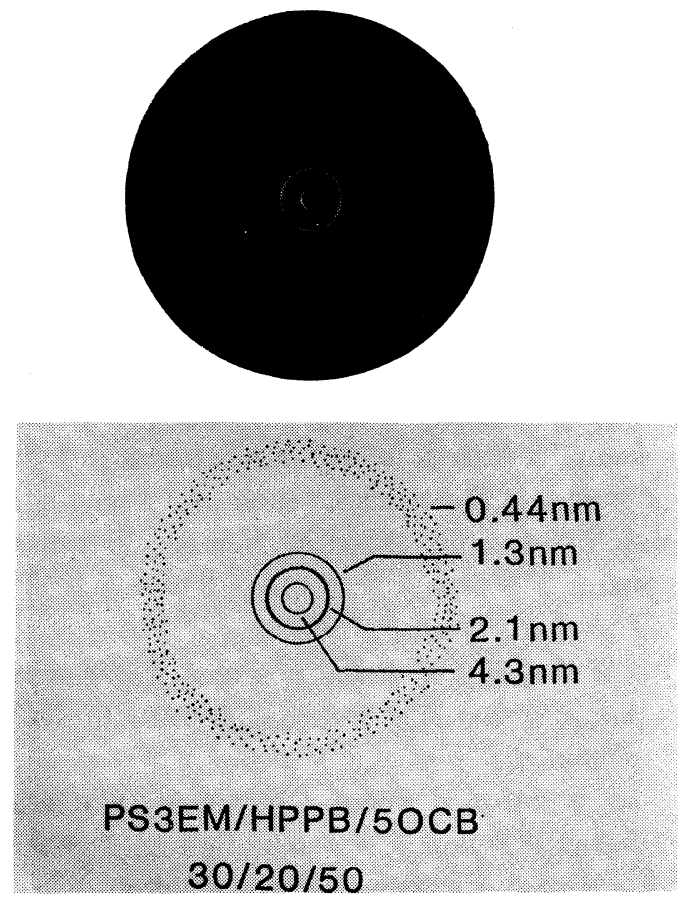

Figure 5. Schematic representation of the X-ray diffraction patterns of the (PS3EM/HPPB/5OCB : 30/20/50 $\mathrm{mol} \%$ ) composite in an induced smectic phase at $329 \mathrm{~K}$.

$1: 2: 3$. The magnitude of most inner diffraction corresponds to roughly twice as long as the mesomorphic side chain length of PS3EM of $2.16 \mathrm{~nm}$. A diffuse reflection of about $0.44 \mathrm{~nm}$ corresponds to an inter-chain or intermolecular distance in the mesogenic side chain group, being composed of the side chain of PS3EM, and HPPB or 5OCB molecules. The sharp $\mathrm{X}$-ray diffraction rings from an induced smectic phase were observed for the (PS3EM/HPPB/ 5OCB : $50-X / X / 50)$ ternary composite system with the whole range of HPPB $(0 \leq X \leq 50)$.

\section{Electro-Optical Effects of the (PS3EM/HPPB/ $5 O C B)$ Composite in an Induced Smectic Phase}

The electro-optical effect based on light scattering was investigated under various conditions of an a.c. electric field. The transmittance of $\mathrm{He}-\mathrm{Ne}$ laser through the composite cell without any optical polarizers 


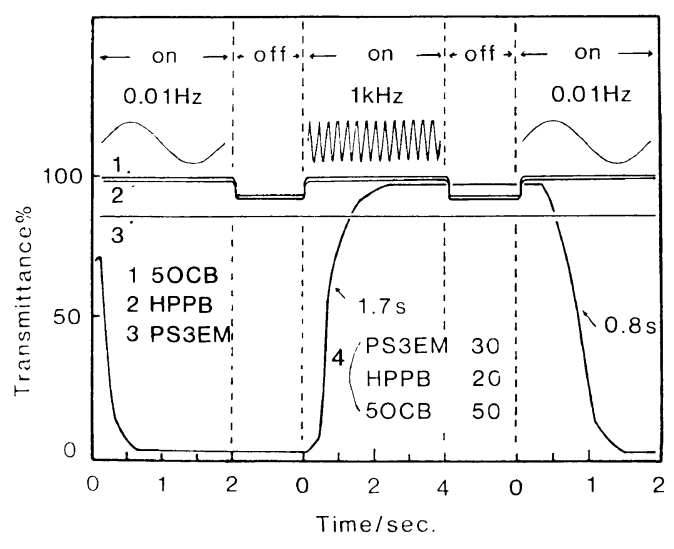

Figure 6. Electro-optical effect of PS3EM (nematic), HPPB (nematic), 5OCB (nematic), and the PS3EM/HPPB/ 5 OCB composite $(30 / 20 / 50 \mathrm{~mol} \%)$ in an induced smectic state upon a.c. electric fields with low $(0.01 \mathrm{~Hz})$ and high $(1 \mathrm{kHz})$ frequencies under the conditions of an applied electric field of $16.7 \mathrm{MV}_{\mathrm{p}-\mathrm{p}} \mathrm{m}^{-1}$, at $T-T_{\mathrm{NI}}=-45 \mathrm{~K}$ (nearly room temperature) and the cell thickness of $16 \mu \mathrm{m}$.

was measured with a photodiode. ${ }^{11}$ Figure 6 shows the transmittances of PS3EM, HPPB, 5OCB, and the (PS3EM/HPPB/5OCB : 30/20/ $50 \mathrm{~mol} \%$ ) composite. In the cases of PS3EM, HPPB, and 5OCB, no distinguishable transmittance changes were observed between a.c. electric field off- and on-states. As shown by the curve 4 in Figure 6 the (PS3EM/HPPB/ 5OCB : $30 / 20 / 50 \mathrm{~mol} \%$ ) composite exhibited a bistable and reversible light switching driven by a.c. electric fields with low and high frequencies. Since the application of a low frequency electric field may induce an ionic current throughout the composite film, it is expected that an induced turbulent flow of LCP polymer main chains, being caused by an ionic current, collapses a fairy well-organized large smectic layer into many small smectic fragments. ${ }^{15,16,21,22}$ That is, there are several possible supports to explain the origin of light scattering for the (LCP/LC) composite system upon the application of an a.c. electric field with low frequency; (1) in the case of low molecular weight LC molecules with a positive dielectric anisotropy in a smectic phase, light scattering cannot be induced with a low electric field, (2) a light scattering state of the (LCP/LC) composite is more enhanced with a larger fraction or a bigger dimension of ionic materials and also, (3) an effective purification of LCP and LC reduces a light scattering effect. Therefore, it is reasonable to consider that the random orientation of smectic directors due to a turbulent flow of polymeric main chains induced by an ionic current can be one of origins for optical heterogeneity to generate strong light scattering. On the other hand, the light transmittance increased up to $99 \%$ upon the application of a high frequency electric field. Any ionic current is not induced upon the application of an a.c. electric field with high frequency but a large scale of homeotropic alignment of smectic layers is easily formed due to a positive dielectric anisotropy of the smectic layer being composed of LC molecules and the side chain group of LCP. Also, turbid and transparent states of the (PS3EM/HPPB/ 5OCB) ternary composite system were remained unchanged even though a.c. electric fields with low and high frequencies were removed, respectively as shown in Figure 6. This means that the ternary composite system in an induced smectic phase has a bistable light switching characteristic, that is, a memory effect.

Figure 7 shows the HPPB fraction, $X$ dependence of rise and decay response times, $\tau_{R}$ and $\tau_{D}$ for the (PS3EM/HPPB/5OCB) ternary composite system. The magnitudes of $\tau_{R}$ and $\tau_{\mathrm{D}}$ were effectively decreased to one-several hundredth ms order by an addition of HPPB, especially in the range of $X$ above $20 \mathrm{~mol} \%$ for the (PS3EM/HPPB/5OCB : $50-X / X / 50$ mol\%) composite at $T-T_{\mathrm{NI}}=-45 \mathrm{~K}$ (nearly room temperature). In the case of the binary (PS3EM/5OCB) composite system, $\tau_{R}$ and $\tau_{D}$ were 200 and 120 seconds at room temperature, respectively. ${ }^{23}$ However, as shown in Figure 7, $\tau_{R}$ and $\tau_{D}$ for the (PS3EM/HPPB/5OCB) ternary composite system were much smaller than those for the binary composite system under the corresponding experimental condi- 


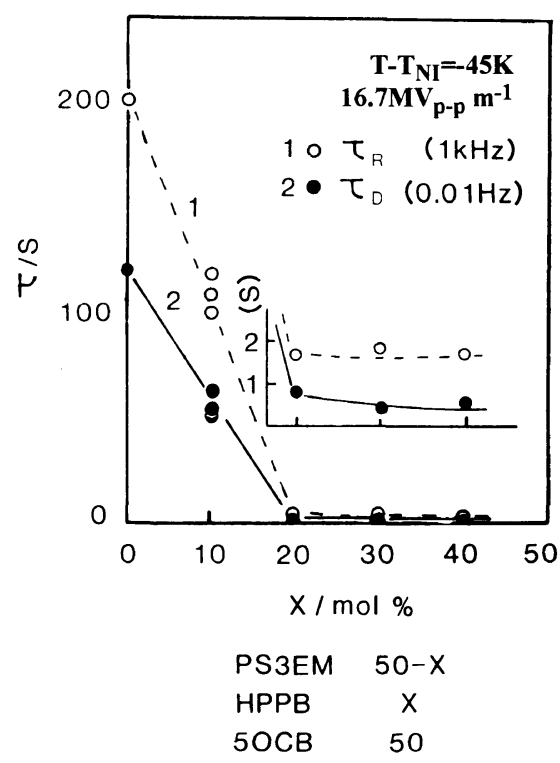

Figure 7. HPPB molar fraction dependence of $\tau_{\mathbf{R}}$ and $\tau_{\mathrm{D}}$ for the (PS3EM/HPPB/5OCB : $50-X / X / 50$, mol\%) ternary composite system under the same conditions in Figure 6.

tions, that is, the same reduced temperature $\left(T-T_{\mathrm{NI}}=-45 \mathrm{~K}\right)$ and the same magnitude of an electric field $\left(16.7 \mathrm{MV}_{\mathrm{p}-\mathrm{p}} \mathrm{m}^{-1}\right)$. Especially, the magnitude of $\tau_{D}$ ranges in several hundred ms even though the composite system has a stable memory effect at room temperature. Therefore, Figure 7 shows that the response speed for bistable and reversible light switching can be remarkably improved by an introduction of HPPB as the third component to the binary (PS3EM/5OCB) composite system in an induced smectic phase, since an addition of HPPB reduces effectively the magnitude of viscosity in the composite system.

\section{CONCLUSION}

The ternary composite was prepared from a miscible mixture of nematic PS3EM with a weak polar terminal unit in the mesogenic side group, nematic 5OCB with a strong polar cyano end group and HPPB with no such a group. An induced smectic phase was observed over a wide range of both mixing concentration and temperature. The mol\% of PS3EM can be reduced by mixing the third component of HPPB, of which chemical structure is similar to the side chain mesogenic group of PS3EM. The (PS3EM/HPPB/5OCB) ternary composite system can maintain the similar characteristics of an induced smectic phase to the (PS3EM/ 5OCB) binary one. The reversible and bistable turbid-transparent change was successfully realized for the binary and ternary composites in an induced smectic phase upon the application of a.c. electric fields with low and high frequencies, respectively. The transparent and turbid states were maintained stably (memory), even though an electric field was turned off. The random orientation of smectic directors due to a turbulent flow of polymeric main chains being induced by an ionic current can be one of origins for optical heterogeneity to generate strong light scattering. It is reasonably concluded that a bistable and reversible light switching of the (PS3EM/ HPPB/5OCB) ternary composite system in an induced smectic phase can be realized by the balance between an electric current effect based on the electrohydrodynamic motion of the LCP main chain and an electric field effect based on the dielectric anisotropy of the smectic layer being composed of LC molecules and the side chain part of LCP. The response speed for bistable and reversible light switching could be remarkably improved by an introduction of HPPB. The magnitudes of $\tau_{R}$ and $\tau_{D}$ are a few seconds and several hundred ms, respectively.

\section{REFERENCES}

1. T. Kajiyama, Y. Nagata, E. Maemura, and M. Takayanagi, Chem. Lett., 679 (1979).

2. S. Washizu, I. Terada, T. Kajiyama, and $\mathbf{M}$. Takayanagi, Polym. J., 16, 307 (1984).

3. H. Kikuchi, A. Kumano, T. Kajiyama, M. Takayanagi, and S. Shinkai, J. Chem. Soc. Jpn., Chem. Ind. Chem., 423 (1987).

4. H. G. Craighead, J. Cheng, and S. Hwackwood, Appl. Rhys. Lett., 40, 22 (1982). 
5. J. L. Fergason, SID Int. Symp. Dig. Tech., 16, 68 (1985).

6. H. Kikuchi, A. Miyamoto, A. Takahara, T. Furukawa, and T. Kajiyama, Preprints, 2nd SPSJ International Polymer Conference, The Society of Polymer Science, Japan, Tokyo, 1986, p. 33.

7. P. S. Drazic, J. Appl. Phys., 60, 2142 (1986).

8. J. W. Doane, A. Golemme, J. L. West, J. B. Whiteneat, and B-G. Wu, Mol. Cryst. Liquid Cryst., 165, 511 (1988).

9. T. Kajiyama, A. Miyamoto, H. Kikuchi, and Y. Morimura, Chem. Lett., 1989, 813 (1989).

10. T. Kajiyama, H. Kikuchi, A. Miyamoto, and Y. Morimura, "Frontiers of Macromolecular Science", Proceedings of the IUPAC 32nd International Symposium on Macromolecules, Blackwell Scientific Publication (UK), 1989, p 505.

11. A. Miyamoto, H.Kikuchi, Y. Morimura, and T. Kajiyama, New Polym. Mater., 2, 1 (1990).

12. C. B. McArdle, Ed., "Side Chain Liquid Crystal Polymers," Chapman and Hall, Inc., New York, N. Y., 1989.

13. A. I. Hopwood and H. J. Coles, Polymer, 26, 1312
(1985).

14. M. S. Sefton and H. J. Coles, Polymer, 26, 1319 (1985).

15. T. Kajiyama, H. Kikuchi, A. Miyamoto, S. Moritomi, and J. C. Hwang, Chem. Lett., 817 (1989).

16. H. Kikuchi, J. C. Hwang, and T. Kajiyama, Polym. Adv. Technol., 1, 297 (1991).

17. A. C. Griffin and J. F. Johnson, J. Am. Chem. Soc., 99, 4859 (1977).

18. B. Engelen, G. Heppke, R. Hopf, and F. Schnider, Ann. Phys., 3, 403 (1978).

19. M. Domon and J. Billard, J. Phys., Paris, 40, C3-413 (1979).

20. F. Schneider and N. K. Sharna, Z. Naturforsch., 36, 62 (1981).

21. T. Kajiyama, H. Kikuchi, A. Miyamoto, S. Moritomi, and J. C. Hwang, Mater. Res. Soc. Sym. Proc., 171, 305 (1990).

22. T. Kajiyama, H. Kikuchi, J. C. Hwang, A. Miyamoto, S. Moritomi, and Y. Morimura, Prog. in Pacific Polym. Sci., 1, 343 (1991).

23. J. C. Hwang, H. Kikuchi, and T. Kajiyama, Polymer, 33, 1821 (1992). 